\title{
Revisiting the role of magnetic moments in heliospheric plasmas
}

\author{
H.-J. Fahr and M. Siewert
}

\begin{abstract}
Argelander Institut für Astronomie der Universität Bonn, Abteilung f. Astrophysik und Extraterrestrische Forschung, Auf dem Huegel 71, 53121 Bonn, Germany

e-mail: msiewert@astro.uni-bonn.de
\end{abstract}

Received 20 December 2012 / Accepted 14 February 2013

\begin{abstract}
The magnetic moment is one of the most fundamental, nontrivial particle properties known in plasma physics. Also known as the first adiabatic invariant, it is conserved under a broad range of microphysical processes and plasma boundary conditions. However, especially in modern numerical simulations, the conservation of this property, which in principle may serve as a control parameter, is no longer used explicitly. In light of this fact, as well as in a recent series of studies of the solar wind termination shock and comparable systems, where this conservation was used as a key ingredient, we now revisit this old, but standing problem. Building on our earlier arguments on when the magnetic moment of individual particles is conserved, we now carefully expand this argument further, studying nontrivial systems, such as systems where individual ions are represented by a broad distribution function that is described with the help of a transport equation. We see that the magnetic moment is conserved under an even wider variety of situations than those studied in earlier publications. When studying systems of purely kinetic equations, the magnetic moment can be converted into an additional force term in the transport equation. We also study the physics that is taken into account in shock simulations and pinpoint weaknesses in the physics used by these modern codes.
\end{abstract}

Key words. plasmas - Sun: heliosphere

\section{Introduction}

One of the most known invariants identified in plasma theory is the individual ion/electron (scalar) magnetic moment

$\mu=\frac{m v_{\perp}^{2}}{2 B}=$ const.

where $v_{\perp}$ is the particle velocity component perpendicular to the magnetic field $B$, and $m$ it's mass. (In the following, we will only talk about ions, dropping the separate notation for electrons.) This property essentially describes the motion of an individual ion gyrating around a background magnetic field (see e.g. Chen 1977). Assuming that the motion of the ion can be averaged over a gyroperiod, resulting in a quasi-stationary continuous electric current density $\boldsymbol{j}(\boldsymbol{r})$, this current will generate a magnetic field that can be developed in a magnetic multipole development, where the first nonvanishing moment is the magnetic dipole moment $\boldsymbol{\mu}$, which does not depend on the charge of the gyrating ion; the absolute value of this vector then is the magnetic moment (Eq. (1)).

Also known as the first adiabatic invariant, the magnetic moment is known to be conserved when external physical parameters (i.e. in the case of the magnetic moment, the background magnetic field $B$ ) of the system are changing sufficiently slowly, so that the motion of the isolated ion, when averaged over a gyration period $\tau_{\mathrm{g}}$, can be smeared out to a continuous circle current. An additional requirement for the validity of adiabatic invariants is the absence of efficient stochastic processes, such as particleparticle and particle-wave scattering. However, even though under these conditions, no strict conservation of $\mu$ can be expected, the basic magnetic Lorentz force still favors a gyrational motion, and therefore, the final transport equation describing the system must still be formulated using a term describing the tendency of the magnetic moment conservation, which operates independent of the convection and diffusion terms (see Sect. 3).

In most astrophysical plasma systems, the ion's density is sufficiently small that binary collisional particle-particle scattering is inefficient, while plasma-wave interactions can often not be ruled out. Therefore, it is not surprizing that the (in-)validity of $\mu$-conservation is a somewhat controversial point in the plasma literature of the past. Though nowadays it is expected that modern MHD shock simulations of the solar wind termination shock (see e.g. Li \& Zank 2006; Kucharek et al. 2006, 2010; Giacalone \& Jokipii 2006) may suggest that ions under such conditions do not conserve their initial magnetic moment, many different conclusions opposite to that point have been achieved in earlier and alternative shock literature. If, for example, one studies the paper by Sarris \& van Allen (1974), one finds that these authors successfully represent, by theoretical calculations, data on shock-processed ions near the Earth's bowshock observationally obtained by the satellites Explorer 33 and 35. As these authors can show a fairly good fit to the observational data is obtained when assuming that these particles are multiply reflected at their magnetic mirror points and finally are moving downstream of the shock with an energy gain corresponding to $v_{2}^{2} / v_{1}^{2} \simeq B_{2} / B_{1}$ (indices are marking upstream (1) and downstream (2) values of particle velocity and magnetic field magnitude, respectively). This result, in optimal accordance with the observed ion data, proves directly that the magnetic moments of these particles while crossing over the shock is in fact conserved.

A similar conclusion is reached by Terasawa (1979) who compares ion spectra of MHD shock-reflected ions calculated by two different approaches, namely the "adiabatic approach" and the so-called "kink treatment". Here the first approach automatically should apply to the situation when the shock structure is 
large with respect to the ion gyroradius and then evidently conserves the ion magnetic moment, while the kink-field approach applies to the case when the shock structure is small with respect to the ion gyroradius. Interestingly enough the results for the spectra of the reflected ions are nearly identical in both cases, meaning that, surprizingly enough, in both cases ions behave, as if their individual magnetic moments are conserved independent on the extent of the shock transition region.

On the other hand people have hesitations to accept the formulation of a tendency to conserve the ion magnetic moment in transport equations, especially if - in addition to conservative forces - collisions or wave-particle interactions operate which at the same time tend to not conserve this quantity. For instance, Barakat \& Schunk $(1983,1989)$ had developed a model of the ion distribution in the cusp region of the geomagnetic field in which they used kinetic theory under the restriction of conserved total ion energy and ion magnetic moment. This approach then later has been enlarged by Barghouthi et al. (1994) and Barakat \& Barghouthi (1994) by including wave-particle interactions in the form of Fokker-Planck type diffusion terms describing a diffusion in the velocity component perpendicular to $\boldsymbol{B}$. Similarly, Le Roux \& Webb (2006) have carried out a test particle calculation solving the transport equation at the solar wind termination shock. In this equation they use, on the left side of the equation, a Liouville operator which takes care of energy and magnetic moment conservation at free ion motions, and at the same time on the right side use a Fokker-Planck diffusion term for pitch-angle scattering. While the latter process definitely violates magnetic moment conservation, in the full transport equation it is nevertheless required to have the correct operation of a Liouville operator that guarantees the conservation of particle invariants in the case of absence or weakness of other stochastic processes. This is the reason why also recently Fahr \& Fichtner (2011), in their recent treatment of pick-up ion transport, do include terms to describe both the tendency to conserve the magnetic moment and Fokker-Planck terms to describe velocity space diffusion.

Finally, some more recent analytical studies do, again, make explicit use of the conservation of the magnetic moment. Based on a collisionless (and, until now, diffusionless) transport equation, Fahr \& Siewert (2006); Siewert \& Fahr (2008) have made extensive use of the magnetic moment conservation to derive an effective force term acting on individual ions crossing an astrophysical shock wave, and found an analytical relation between upstream and downstream distribution functions. With the same assumptions, they treated the processing in velocity space of an ion distribution function undergoing multiple passages of travelling shock fronts (Fahr et al. 2012a). Intermixed with strong isotropisation processes, they discovered a mechanism that allows to generate stable power law distribution functions, which have in fact been observationally found in most astrophysical systems. Finally, they also studied an electrostatic beam instability as a mechanism violating conservation of the magnetic moment in multicomponent systems Verscharen \& Fahr (2008); Fahr et al. (2012b).

Due to the broad range of these examples (analytical modeling, numerical modeling, and also inspection of space plasma data), it is obvious that the conservation of the magnetic moment is a highly nontrivial question which, until now, has never been studied explicitly and ab initio with sufficient detail and generality. The only recent, related study on magnetic moments in plasma systems was presented by Cary \& Brizard (2009), who derived a Hamiltonian formulation of the classical guiding center theory, however without going into practical details or reaching a definite conclusion when the magnetic moment is conserved. This study here aims to close this gap in theory using a different approach, collecting and expanding existing arguments on a more general base. We demonstrate several counterintuitive properties of the magnetic moment and derive hard criteria that allow to reevaluate existing models, and also to test existing numerical implementations with respect to their physical validity.

Our approach to this problem is as follows. First, we will present general properties of the magnetic moment, as they are found with varying degree of accuracy in existing textbooks, pointing out those properties that are basic for understanding non-textbook scenarios. Following this, we will study these nonideal systems, where some of the general assumptions commonly found in literature are relaxed, or even dropped. In the section following this one (Sect. 3), we carefully study the role of magnetic moment conservation in transport equations, with an emphasis on when it makes sense to add an explicit term to the equations to respect the magnetic moment physics, and when this term is already, implicitly, present. Finally, in the conclusions, we briefly touch the wide field of numerical simulations, studying how our current results will allow to estimate the behavior of existing (and possibly upcoming) codes with respect to the conservation of the magnetic moment.

\section{The magnetic moment of individual ions in a plasma}

In the following sections, we study old and new aspects of the conservation of an individual ion's magnetic moment in a plasma in the test particle approach, i.e. where test ions are moving in a predetermined electromagnetic field generated by independent parts of the plasma.

The historical reason why Eq. (1) is called the magnetic moment of the ion (or likewise the electron) is due to the fact that the gyrational motion of the ion in fact represents an electric circle current $\boldsymbol{j}(\boldsymbol{x})$ that acts in an electromagnetic sense like a magnetic dipole $\mu_{D}$ given by

$\mu_{\mathrm{D}}=-\frac{1}{c} \frac{e \omega_{\mathrm{g}}}{2 \pi}\left(\pi r_{\mathrm{g}}^{2}\right)(\boldsymbol{B} / B)=-\mu_{B}(\boldsymbol{B} / B)$,

where $\omega_{\mathrm{g}}$ is the gyration frequency, $r_{\mathrm{g}}$ is the gyration radius, and $B$ the background magnetic field strength. Since this magnetic moment $\mu_{\mathrm{D}}$ is always directed opposite to the main field $\boldsymbol{B}$, ions (and electrons as well) contribute a diamagnetic action in a magnetized plasma. In plasma physics, especially in test particle approaches, one is often interested in the dynamic properties of an individual ion, the test particle, instead of the averaged properties of the entire system. This test ion typically is sent into a predefined region of arbitrarily complicated magnetic and electric fields, and the individual ion's scalar magnetic moment $\mu$ (see Eq. (1)) is sometimes used as a control parameter. However, studying the motion of an arbitrary ion is violating the requirements of magnetostatics as defined in the previous section, as the individual ion will be located at different positions at different times, usually not generating a quasistationary electric current, not even in the line-convected frame.

Therefore, we need to apply a specific average, the gyrolimit, to convert our time-dependent individual ion motion into a quasistationary, time-independent current density $\boldsymbol{j}(\boldsymbol{x})$, confined to a sufficiently small region of space, "small" being defined as small compared to any length scale of interest to an observer. The degree of details given on this approximation varies greatly in literature, which is why we now repeat the most relevant aspects. First, it is possible to perform a multipole expansion in 
terms of vector spherical harmonics of the magnetic vector potential $\boldsymbol{A}(\boldsymbol{x})$, which can be derived from $\boldsymbol{j}(\boldsymbol{x})$. For a localized, divergence-free current density, the first nontrivial, nonvanishing term in the mentioned multipole expansion is given by (see Jackson 1975)

$\boldsymbol{A}=\frac{\boldsymbol{\mu} \times \boldsymbol{x}}{x^{3}}$,

where $\boldsymbol{\mu}$ is the development coefficient called the magnetic moment, which is defined by

$\boldsymbol{\mu}=\frac{1}{2} \int \mathrm{d}^{3} x^{\prime} \boldsymbol{x}^{\prime} \times \boldsymbol{j}\left(\boldsymbol{x}^{\prime}\right)$.

This equation, which just represents a development coefficient, is often found as the definition of the magnetic moment. For an arbitrary charged current system, this vector usually is not an invariant. However, in the magnetostatic limit, i.e. for $\frac{\mathrm{d}}{\mathrm{d} t} \boldsymbol{j}=0$, it is easy to demonstrate that the above integral usually reduces to a constant value. It should be pointed out here that this approach to define a magnetic moment is not restricted to magnetostatic situations, though; in principle, it would be possible to define a "dynamic" magnetic moment by taking, e.g., the more general retarded vector potential describing a nonstatic system, and define a generalized magnetic moment as the development coefficient appearing in the retarded description. Even though deriving such a generalized magnetic moment would break the limit of this paper, this proves that the basic ideas behind the concept of the magnetic moment could be generalized.

Now, aiming to approximate a stationary system, where the charge currents are time-independent, it is assumed that the ion is performing an unperturbed gyrational motion where the gyroperiod $\tau_{\mathrm{g}}=\omega_{\mathrm{g}}^{-1}$ is much smaller than all other timescales of interest, including the timescale for background field changes $\left(\tau_{B}=\dot{B} / B\right)$. In this case, it is possible to average the motion of the individual ion over one gyroperiod, i.e. to derive a quasistationary circle current, $\langle\boldsymbol{j}\rangle$, which no longer depends on time and allows to derive a magnetic moment consistent with the one used in plasma theory (i.e. with an absolute value identical to Eq. (1)).

Using such an averaged stationary electric current, Eq. (4) transforms into

$\boldsymbol{\mu}=q \frac{1}{4 \pi} \int \mathrm{d} \phi \boldsymbol{r}_{\mathrm{g}}(\phi) \times \boldsymbol{v}(\phi)$,

where we integrate over one gyrocircle, using polar coordinates (i.e. $\boldsymbol{r}_{\mathrm{g}}(\phi)=r_{\mathrm{g}}(\cos \phi, \sin \phi, 0)$, and $\left.\boldsymbol{v}(\phi)=\frac{\mathrm{d}}{\mathrm{d} t} \boldsymbol{r}_{\mathrm{g}}\right)$. Evaluating this integral is trivial, and immediately leads to Eq. (1). Assuming that the motion of the gyration is located in a plane (i.e. the motion is 2-dimensional, as for the gyrating ion discussed above), and that the current loop is closed (but not necessarily a circle), then the scalar magnetic moment is given by (see e.g. Northrop 1963; Jackson 1975; Cary \& Brizard 2009)

$\mu=I \cdot S$,

where $I=\boldsymbol{j} \cdot \mathrm{d} \sigma$ is the electric current and $S$ the enclosed surface. Therefore, the magnetic moment is conserved for an ion following a fixed planar motion, in the magnetostatic gyroaverage limit, due to the fact that both the electric current and the enclosed surface remain constant.

\subsection{The diamagnetic moment of the magnetized plasma}

The magnetic moment defined by Eq. (2) is a single-particle formulation of the more general magnetisation $\boldsymbol{M}$, i.e. the magnetic moment of the plasma per volume unit. In the continuous plasma formulation, the relation between the (global) magnetic field and the magnetisation is given by

$\boldsymbol{B}=\boldsymbol{H}+4 \pi \boldsymbol{M}$,

which correctly differentiates between the magnetic induction $\boldsymbol{B}$ and the vacuum magnetic field $\boldsymbol{H}$. The magnetic moment of the plasma per volume $\boldsymbol{M}$ is then defined by (see e.g. Fahr \& Scherer 2005)

$\boldsymbol{M}=-\frac{\boldsymbol{B}}{B^{2}} \sum_{i} m_{i} \int f_{i} v_{\perp}^{2} \mathrm{~d}^{3} v$.

The summation runs over the different species $i$ of plasma particles with different masses and distribution functions. While in the inner heliosphere the contributions to $\boldsymbol{M}$ from Maxwellian distributed electrons and protons of the solar wind may be fairly comparable $\left(m_{\mathrm{i}}\left\langle v_{\perp}^{2}\right\rangle_{\mathrm{i}} \simeq m_{\mathrm{e}}\left\langle v_{\perp}^{2}\right\rangle_{\mathrm{e}}\right)$ and relatively small, the Kappa-distributed pick-up ions due to their dominant pressure $P_{\text {pui }}$ most likely contribute the major portion given by (see Fahr \& Scherer 2004)

$\boldsymbol{M}=-\frac{\boldsymbol{B}}{2 B^{2}} P_{\text {pui }}$.

While this diamagnetic behavior of a broad multispecies plasma distribution function is understood in theory, no use is made of its consequences.

\subsection{Slowly varying magnetic fields}

Next, we study the reaction of a gyrating ion to a slowly changing background magnetic field strength, while keeping the orientation of $\boldsymbol{B}$ fixed, i.e. $\dot{\boldsymbol{B}} \| \boldsymbol{B}$ and making use of the gyroaverage introduced above. In addition, it follows from basic electromagnetic concepts that the gyroradius is given by

$r_{\mathrm{g}}=\frac{m v_{\perp} c}{q B}$

Using the assumptions on the magnetic field introduced above, this parameter $r_{\mathrm{g}}$ is no longer constant due to $\dot{B} \neq 0$, and also due to induced electric forces modifying $v_{\perp}$ (as we will demonstrate further down). Therefore, the enclosed surface will change accordingly - but also the electric current strength $I$ flowing along the border line of the enclosed surface. For a line-like current due to an electric charge circling a gyrocircle at constant speed $v_{\perp}$ over one gyroperiod, the current strength is given by

$I=|\boldsymbol{j}|=\frac{q}{l} v_{\perp}=\frac{q}{\tau_{\mathrm{g}}}=q \Omega_{\mathrm{g}}$,

where $l$ is the length of the enclosed surface's border line. Then, the magnetic moment as defined by the enclosed surface relation (see Eq. (6)) is given by

$\mu=\frac{q v_{\perp} A}{l}$,

which is only conserved if $\frac{v_{\perp} A}{l}$ is constant. For an arbitrary motion, e.g. of ions moving in strongly inhomogeneous magnetic fields, this relation will not be fulfilled. In fact, if the magnetic field changes over a time scale that is much smaller than the gyrofrequency, the ion will not undergo a closed gyrational motion, and the concept of a constant enclosed surface breaks down.

In the gyrolimit, however, the magnetic moment turns out as

$\mu=\frac{q v_{\perp} \pi r_{\mathrm{g}}^{2}}{2 \pi g_{r}}=\frac{q v_{\perp} r_{\mathrm{g}}}{2}=\frac{m v_{\perp}^{2}}{2 B}$, 
which is the same as the relation introduced earlier in Eq. (1). Differentiating this expression with respect to time, setting the time derivative of $\mu$ to zero with some elementary algebra then results in the following condition:

$m \dot{v}_{\perp}=\frac{m v_{\perp}}{2 B} \frac{\partial B}{\partial t}=\frac{q}{2} r_{\mathrm{g}} \frac{\partial B}{\partial t}$.

The force providing just the correct magnitude and orientation follows from Faraday's law of induction,

$\boldsymbol{\nabla} \times \boldsymbol{E}_{\text {ind }}=-\frac{\partial \boldsymbol{B}}{\partial t}$,

which, after making use of Stoke's theorem, trivially results in the induced electric force (Fahr \& Siewert 2010)

$m \dot{v}_{\perp}=-q E_{\text {ind }}=\frac{q}{2} r_{\mathrm{g}} \frac{\mathrm{d} B}{\mathrm{~d} t}$,

which is just the force required to conserve the magnetic moment.

One might perhaps suspect that, in these above considerations, the influence of a secondary electric field component has been neglected which is due to the temporal change of the vector potential $\boldsymbol{A}$. However, as one can easily demonstrate, this contribution does not influence the gyrating ion if external electric currents can be neglected, since then,

$\mathrm{d} s_{\perp} \cdot \frac{\partial A}{\mathrm{~d} t}=\frac{\partial}{\partial t}\left(\mathrm{~d} s_{\perp} \cdot \int \frac{j^{\prime}}{r^{\prime}} \mathrm{d} V^{\prime}\right)=0$.

\subsection{The magnetic moment and the angular momentum}

Before finishing our introduction on established concepts related to the magnetic moment, we point out one more useful property. Respecting, again, that the ion performs a gyrational motion, the integrand appearing in Eq. (5) is proportional to the orbital angular momentum $\boldsymbol{L}$ with respect to the gyrocenter,

$\boldsymbol{\mu}=\frac{q}{2 m} \frac{1}{2 \pi} \int \mathrm{d} \phi \boldsymbol{L}(\phi)=\frac{q}{2 m}\langle\boldsymbol{L}(\phi)\rangle_{\phi}=\frac{q}{2 m c} \boldsymbol{L}$.

Therefore, the magnetic moment is conserved exactly when the angular momentum is, providing another aspect to our study.

Now, it is trivial to see that the angular momentum is conserved exactly when the torque $\tau$ vanishes,

$$
\begin{aligned}
\boldsymbol{\tau} & =\dot{\boldsymbol{L}} \\
& =m \dot{\boldsymbol{r}}_{\mathrm{g}} \times \boldsymbol{v}+m \boldsymbol{r}_{\mathrm{g}} \times \dot{\boldsymbol{v}},
\end{aligned}
$$

where the first term is associated with a force reducing the gyroradius. In most (mechanical) textbooks, this term is argued away by selecting a specific, center-of-mass reference frame that allows to keep the distance of the rotating mass points constant. However, examples exist for systems where the angular momentum is conserved even though the radius of the circular motion with respect to it's center is not constant. Therefore, this first term is actually important for understanding the general behavior of the angular momentum, and therefore, the magnetic moment.

To further elaborate this claim, we now derive the classical torque acting on a gyrating ion in the presence of time-dependent magnetic fields. The induced electric force derived in the previous section generates a nonzero torque acting on the gyrating ion, which is of the magnitude

$\boldsymbol{\tau}=\frac{m}{e} \boldsymbol{r}_{\mathrm{g}} \times \boldsymbol{E}_{\text {ind }} \propto \boldsymbol{e}_{x} \times \boldsymbol{e}_{v} \neq 0$, where $\boldsymbol{e}_{i}$ are unit vectors pointing into the direction of the subscript vector $i$ (e.g. $\left.\boldsymbol{e}_{B}=\boldsymbol{B} /|B|\right)$. This equation proves that the induced electric force modifies $v_{\perp}$, the vector product between $\boldsymbol{r}_{\mathrm{g}}$ and $\boldsymbol{E}_{\text {ind }}$ does not vanish, it seems like the torque is nonzero, and the angular momentum seems to be not conserved.

However, one also has to consider that the gyroradius $r_{\mathrm{g}}$, which is a function of $v_{\perp}$ and $B$ (see Eq. (10)), is not constant either, which, effectively, results in an additional force acting on the gyrating ion. This force, which results in

$\dot{x}=\dot{\boldsymbol{r}}_{\mathrm{g}} \neq 0$,

contributes to the first term in the definition of the torque (Eq. (19)). As it is trivial to verify, this term compensates the classical torque, allowing to arrive at a conserved angular momentum. This demonstrates that the identification of the magnetic moment $\mu$ with the angular momentum $\boldsymbol{L}$ is nontrivial, and a consistent description of gyrating ions requires a full treatment of electromagnetic effects.

\subsection{Weakly spatially inhomogeneous magnetic fields}

In general, a magnetic field will never be as ideal as assumed in our previous examples, but undergo local variations and fluctuations. Omitting an explicit time-dependence, the commonly found parameterisation of this behavior is given by

$\boldsymbol{B}(\boldsymbol{x})=\boldsymbol{B}_{0}+\Delta \boldsymbol{B}(\boldsymbol{x})$,

where our previous examples are characterized by $\Delta \boldsymbol{B} \equiv 0$ over the entire relevant spatial region. In a turbulent system dominated e.g. by plasma waves, dipolar/multipolar fields or even just in case of the solar wind magnetic field dropping in intensity with solar distance, this will no longer be the case. For example, when the wavelength of a plasma wave is on the order of the gyroradius, the magnetic field inducing the gyrational motion may no longer be conserved.

We begin our study of this configuration by assuming a static inhomogenity, where the background magnetic field is defined by a magnetostatic, but not spatially constant $(\Delta \boldsymbol{B} \neq 0)$ configuration where, over one gyroperiod, the ion at its gyration is experiencing slightly different magnetic field strengths. Without loss of generality, we now select a coordinate system where $\boldsymbol{B}_{0} \| \boldsymbol{e}_{z}$, i.e. the gyrational motion will be initially confined to the $x y$ plane. In addition, we assume that $\frac{\partial B_{z}}{\partial z} \neq 0$, i.e. that a linear spatial gradient into the $z$-direction exists, i.e. $\Delta \boldsymbol{B}(\boldsymbol{x})$ is a linear function in $\boldsymbol{x}$ that is small compared to $B_{0}$. In addition, it follows from the source-freeness of the magnetic field that $\boldsymbol{B}(\boldsymbol{x})$ must be divergence-free, i.e.

$\frac{\partial B_{x}}{\partial x}+\frac{\partial B_{y}}{\partial y}=-\frac{\partial B_{z}}{\partial z}$.

Then, it follows from Eq. (23) that a magnetic field gradient in the $z$-direction automatically induces an additional magnetic field component in the $x y$-plane, which generates a force that depends on the gyroangle $\phi$,

$\boldsymbol{F}_{\perp}=\frac{q}{c} \boldsymbol{v}_{\perp}(\phi) \times \Delta \boldsymbol{B}_{\perp}(\phi)$.

This force is oriented parallel to the $z$ direction and possesses an absolute magnitude of

$$
\begin{aligned}
F_{z} & =\frac{q}{c}\left(v_{x} \Delta B_{y}-v_{y} \Delta B_{x}\right) \\
& =\frac{q}{c}\left(v_{x} r_{y} \frac{\partial B_{y}}{\partial y}-v_{y} r_{x} \frac{\partial B_{x}}{\partial x}\right) .
\end{aligned}
$$


Now, inserting the usual expressions for a circular motion $\left(r_{x}=\right.$ $r_{\mathrm{g}} \cos \phi, r_{y}=r_{\mathrm{g}} \sin \phi, v_{x}=-v_{\perp} \sin \phi$ and $\left.v_{y}=v_{\perp} \cos \phi\right)$, we obtain

$F_{z}=-\frac{q}{c} v_{\perp} r_{\mathrm{g}}\left(\sin ^{2} \phi \frac{\partial B_{y}}{\partial y}+\cos ^{2} \phi \frac{\partial B_{x}}{\partial x}\right)$

Averaging this over $\phi$, i.e. over one full gyroperiod, we obtain

$$
\begin{aligned}
F_{z} & =-\frac{q}{2} v_{\perp} r_{\mathrm{g}}\left(\frac{\partial B_{y}}{\partial y}+\frac{\partial B_{x}}{\partial x}\right) \\
& =\frac{1}{2} \frac{m v_{\perp}^{2}}{B} \frac{\partial B_{z}}{\partial z} \\
& =\mu \frac{\partial B_{z}}{\partial z} .
\end{aligned}
$$

In other words, the (average) force is proportional to the magnetic moment and the spatial field gradient into the $z$-direction, and it is oriented parallel to the magnetic field.

This force, which does not change the full kinetic energy of the particle due to $\mathrm{d} v_{\|}^{2}=-\mathrm{d} v_{\perp}^{2}$, allows us to solve the equation of motion parallel to $B$ by

$$
\begin{aligned}
m \frac{\mathrm{d} v_{\|}}{\mathrm{d} t} & =m v_{\|} \frac{\mathrm{d} v_{\|}}{\mathrm{d} z} \\
& =\frac{\mathrm{d}}{\mathrm{d} z}\left(\frac{m}{2} v_{\|}^{2}\right) \\
& =-\frac{\mathrm{d}}{\mathrm{d} z}\left(\frac{m}{2} v_{\perp}^{2}\right) \\
& =F_{z}=-\mu \frac{\mathrm{d} B_{z}}{\mathrm{~d} z} .
\end{aligned}
$$

Obviously, this equation is solved by any solution fulfilling $\mathrm{d}\left(v_{\perp}^{2} / B\right)=0$, which in turn is equivalent to the conservation of the magnetic moment $\mu$. This means that the motion of the gyrating ion along a field line takes place such that $\mu$ is conserved. This result also leads to the well established phenomenon of mirror points and particle reflections at critical fields $B_{\mathrm{c}}=B /\left(1-\xi^{2}\right)$, for particles with pitchangle cosines $\xi$ at a certain point where the field has a strength of $B$.

\subsection{Magnetic induction in the guiding center frame}

Next, we study the conservation of the magnetic moment in a case where, in addition to a time-dependent magnetic field strength, also a reorientation of the magnetic field is introduced. Due to mathematical complications related to transforming local gyrational velocities, we will perform this part of the study in the framework of guiding center theory.

We begin with a variation of previously introduced concepts by introducing the magnetic flux $\Phi=\pi r_{\mathrm{g}}^{2} B$. The temporal derivative in the guiding center rest frame is given by

$\frac{\mathrm{d}}{\mathrm{d} t} \Phi=2 \pi r_{\mathrm{g}} \frac{\mathrm{d} r_{\mathrm{g}}}{\mathrm{d} t} B+\pi r_{\mathrm{g}}^{2} \frac{\mathrm{d} B}{\mathrm{~d} t}$

Obviously, this flux is not constant for an arbitrary behavior of $r_{\mathrm{g}}$ and $B$. For this expression to be constant, we need that

$$
\frac{1}{v_{\perp}} \frac{\mathrm{d} v_{\perp}}{\mathrm{d} t}-\frac{1}{2 B} \frac{\mathrm{d} B}{\mathrm{~d} t}=0
$$

which, again, is a formulation of the requirement that the magnetic moment $\mu=\frac{m v_{\perp}^{2}}{2 B}$ is conserved.
This identification now allows us to study the conservation of the magnetic moment for a system where the magnetic field vector is changing it's orientation, while keeping both $\mu$ and $\Phi$ constant. Assuming that the plane of gyration changes it's orientation so that the local magnetic field vector $\boldsymbol{B}$ is always normal to this plane, this means that the angular momentum $\boldsymbol{L}$ can be written as (see Eq. (18))

$\boldsymbol{L}\left(B, v_{\perp}\right)=\boldsymbol{r}_{\mathrm{g}} \times \boldsymbol{p}=\frac{2 m c}{e} \frac{\mathrm{d}}{\mathrm{d} t} \mu \boldsymbol{e}_{B}$,

where, again, $\boldsymbol{e}_{B}=\boldsymbol{B} /|B|$ is a unit vector. The derivative with respect to time then is given by

$\frac{\mathrm{d}}{\mathrm{d} t} \boldsymbol{L}\left(B, v_{\perp}\right)=\frac{2 m c}{e}\left(\frac{\mathrm{d} \mu}{\mathrm{d} t} \boldsymbol{e}_{B}+\mu \frac{\mathrm{d} \boldsymbol{e}_{B}}{\mathrm{~d} t}\right)$.

If we now repeat the results from earlier sections, namely that the magnetic moment is conserved for $\dot{B} \neq 0$ and $\dot{\boldsymbol{e}}_{B}=0$, the first term in this expression vanishes, and we obtain

$\frac{\mathrm{d}}{\mathrm{d} t} \boldsymbol{L}\left(B, v_{\perp}\right)=\frac{2 m c}{e} \mu \frac{\mathrm{d} \boldsymbol{e}_{B}}{\mathrm{~d} t}=L v_{\|}\left(\boldsymbol{e}_{B} \cdot \boldsymbol{\nabla}\right) \boldsymbol{e}_{B}$.

This means that the angular momentum of the gyrating ion/electron changes with the magnetic line element $s$ as the field itself is doing, so that both vectors $\boldsymbol{B}$ and and $\boldsymbol{L}$ remain parallel to each other, while the ion is propagating along the field line.

This relation now allows to study the conservation of the scalar magnetic moment for an arbitrary magnetic field behavior. If we assume that the first-order correction to the angular momentum is given by

$\boldsymbol{L}(t+\Delta t)=\boldsymbol{L}(t)+\left.\frac{\mathrm{d} \boldsymbol{L}}{\mathrm{d} t}\right|_{t} \Delta t$,

then obviously the scalar angular momentum is conserved when

$|\boldsymbol{L}(t+\Delta t)|=\sqrt{|L|^{2}(t)+\left(\left.\frac{\mathrm{d} \boldsymbol{L}}{\mathrm{d} t}\right|_{t} \Delta t\right)^{2}+\left.2 \boldsymbol{L} \cdot \frac{\mathrm{d} \boldsymbol{L}}{\mathrm{d} t}\right|_{t} \Delta t}$,

which would imply that the scalar magnetic moment is conserved as well. Evaluating this expression is highly nontrivial and probably requires numerical evaluation of the quantities appearing in this expression. Nevertheless, given a specific (dynamic) magnetic field configuration, Eq. (33) allows to study the conservation of the magnetic moment of ions gyrating under these magnetic conditions.

\subsection{Magnetic moment in more general cases}

The cases studied until here are by no means complete; unfortunately, an arbitrarily complicated, inhomogeneous and dymnamic magnetic field can not be studied easily using fundamental electrodynamics. However, before we continue to the complicated field of transport theory, we would like to summarize a few general arguments that can be used easily to study more general systems using different methods than those presented here.

First, the possibility to identify a magnetic moment with an angular momentum provides a promizing tool that can be adapted to test the conservation of the magnetic moment with e.g. numerical codes. However, a full treatment using this test parameter requires a full and correct treatment of all microphysical electromagnetic forces, including those generated by the induction terms in Maxwell's equations, which is a challenging problem even to modern supercomputers. 
The other major tool that should be useful for future studies of a possible magnetic moment conservation is the gyrolimit. Considering that the magnetic moment usually is associated with a quasistationary (gyro-)limit, it is possible to study the averaged conservation of the magnetic moment by considering the gyrolimit. If the forces and related angular torques acting on a gyrating ion vanish when averaged over one gyroperiod, then it is reasonable to claim that the magnetic moment is conserved, on average, as well. This requires a full, consistent treatment of all microphysical forces.

\section{The magnetic moment in transport theory}

\subsection{The Vlasov equation}

Finally, having demonstrated that the magnetic moment is conserved under a large variety of assumptions, and also deriving an improved condition on when it is conserved exactly, we study how the magnetic moment is treated in existing models for the transport of cosmic rays through the heliosphere and the local interstellar medium. Since $\mu$ represents a derived parameter that can be interpreted quite differently, we now need to study how a term associated with the conservation of $\mu$ can be identified in existing transport equation.

First, as an illustrative example, we look into a Vlasow equation that describes the distribution function $f=f\left(z, v_{\|}, v_{\perp}\right)$ as function of coordinates $z, v_{\|}=v \xi$, and $v_{\perp}=v \sqrt{1-\xi^{2}}$, where $\xi=\cos \angle(\boldsymbol{v}, \boldsymbol{B})$. Then, taking into account the force $k_{z}$ due to the inhomogeneity of the $B$-field and all other forces $k_{\|}$parallel to $\boldsymbol{B}$, one finds for the stationary case the following form of a differential equation (see Fahr et al. 1977):

$$
v_{\|} \frac{\partial f}{\partial z}+\frac{1}{m}\left(k_{\|}-\frac{1}{2} \frac{m v_{\perp}^{2}}{B} \frac{\mathrm{d} B_{z}}{\mathrm{~d} z}\right) \frac{\partial f}{\partial v_{\|}}+\frac{v_{\perp} v_{\|}}{2 B} \frac{\mathrm{d} B}{\mathrm{~d} z} \frac{\partial f}{\partial v_{\perp}}=0 .
$$

Here, $k_{\|}$represents all other than magnetic (e.g. electric or gravitational) forces acting parallel to the field $\boldsymbol{B}$. It is interesting to see that in the case of $k_{\|}=0$, this equation immediately reduces to the equation which is used by Siewert \& Fahr (2008) to describe the changing ion distribution function at the plasma passage over the solar wind termination shock. This latter equation was derived under the assumption of the conservation of the magnetic moment, demonstrating that a term that can be identified with this effect can easily appear in a kinetic equation. Even though this term does not necessarily have to be the only force acting on the ion, it nevertheless suggests that a trend towards the conservation of $\mu$ is implicitly present in many applications of transport theory.

\subsection{The Liouville transport operator in a focussed transport equation}

We now study the implicit conservation of the magnetic moment in kinetic transport equations using a more general argument. In papers dealing with ion phasespace transport like those by e.g. Roelof (1969); Earl (1976); Kunstmann (1979); Spangler \& Basart (1981); Le Roux et al. (2001, 2002); Le Roux \& Webb (2007); Schlickeiser \& Shalchi (2008); Schlickeiser (2009) or Litvinenko \& Schlickeiser (2011), a transport operator for focussed particle motions has been applied. This form of a transport description is always associated with the action of the same typical Liouville operator $L^{\leftrightarrows}$ on the particle distribution function $f$ which describes free particle motions along inhomogeneous magnetic guide fields. This operator is of the form

$L^{\leftrightarrows}(f)=\frac{\partial f}{\partial t}+\xi v \frac{\partial f}{\partial z}+\frac{v}{2 L}\left(1-\xi^{2}\right) \frac{\partial f}{\partial \xi}$,

where the distribution function $f=f(z, v, \xi, t)$ depends on the line coordinate $z$, velocity magnitude $v$, pitchangle cosine $\xi=\cos \vartheta$, and the time $t$. $L$ is the inhomogeneity scale of the background magnetic field.

Now it must be noted that, at the absence of collisions or stochastic interactions, the Liouville theorem always requires that the phasespace density of the function $f$, whatever it's form may be, is constant along each particle trajectory $s=s(t)$, meaning that $\mathrm{d} f / \mathrm{d} s=0$ must be valid. It is now interesting to recognize that this will automatically be fulfilled, if the distribution function can be written as a function of particle invariants of the motion. For example, assuming that the distribution function can be written as a function of the kinetic energy $\left(\propto \boldsymbol{v}^{2}\right)$ and the magnetic moment $\mu=m v^{2}\left(1-\xi^{2}\right) / B, f^{i}=f\left(v^{2}, \mu\right)$, the function is constant when both parameters are particle invariants, because of

$\mathrm{d} f^{i} / \mathrm{d} s=\frac{\partial f^{i}}{\partial v^{2}} \frac{\mathrm{d} v^{2}}{\mathrm{~d} s}+\frac{\partial f^{i}}{\partial \mu} \frac{\mathrm{d} \mu}{\mathrm{d} s}=0$.

For motions in stationary magnetic fields, it is evident that due to

$\frac{\mathrm{d}}{\mathrm{d} t} \boldsymbol{v}^{2} \propto \boldsymbol{v} \cdot \frac{\mathrm{d} \boldsymbol{v}}{\mathrm{d} t}=(e / m c)(\boldsymbol{v} \cdot(\boldsymbol{v} x \boldsymbol{B}))=0$,

the kinetic energy is conserved. Furthermore, if the inhomogeneity scale $L=L_{B}$ of the background field is large with respect to the gyroradius $r_{\mathrm{g}}$ or with respect to the gyrolength $v \mu / \Omega_{\mathrm{g}}$, then it can be assumed that also the magnetic moment of the particle is invariant at the motion along the guide field $B$, and the distribution function should then satisfy the Liouville theorem.

Now, we can immediately show below that the above mentioned Liouville operator for stationary fields (Eq. (37)), when applied to functions parameterized as $f^{i}=f\left(v^{2}, \mu\right)$, automatically fulfills the Liouville theorem, i.e. we will demonstrate that, in this case,

$L^{\leftrightarrows}\left(f^{i}\right)=\xi v \frac{\partial f^{i}}{\partial z}+\frac{v}{2 L}\left(1-\xi^{2}\right) \frac{\partial f^{i}}{\partial \xi}=0$

is valid. This is verified easily by expanding the partial derivatives appearing in the operator, because then, the following is evident:

$\frac{\partial f^{i}}{\partial z}=\frac{\partial f^{i}}{\partial B} \frac{\mathrm{d} B}{\mathrm{~d} z}=\frac{\mathrm{d} B}{\mathrm{~d} z} \frac{\partial f^{i}}{\partial \mu} \frac{\partial \mu}{\partial B}=\frac{\mathrm{d} B}{\mathrm{~d} z} \frac{\partial f^{i}}{\partial \mu}\left(-\frac{\mu}{B}\right)$

and

$\frac{\partial f^{i}}{\partial \xi}=\frac{\partial f^{i}}{\partial \mu} \frac{\mathrm{d} \mu}{\mathrm{d} \xi}=\frac{\partial f^{i}}{\partial \mu}\left(-\frac{2 \xi \mu}{\left(1-\xi^{2}\right)}\right)$

Now, we remind the reader that the field inhomogenity length scale $L_{B}$ is defined through $\frac{1}{L_{B}}=-\frac{1}{B} \frac{\mathrm{d} B}{\mathrm{~d} z}$ (see Earl 1976). This allows us to write

$\xi v \frac{\partial f^{i}}{\partial z}+\frac{v}{2 L}\left(1-\xi^{2}\right) \frac{\partial f^{i}}{\partial \xi}$

$=\xi v \frac{\mathrm{d} B}{\mathrm{~d} z} \frac{\partial f^{i}}{\partial \mu}\left(-\frac{\mu}{B}\right)-\frac{v}{2 L}\left(1-\xi^{2}\right) \frac{\partial f^{i}}{\partial \mu} \frac{2 \xi \mu}{\left(1-\xi^{2}\right)}=0$,

and proves our above mentioned claim that the scatter-free Liouville operator used in a transport equation automatically 
and necessarily implies the conservation of the magnetic moment, even when this conservation can not be concluded from a suffiently long inhomogenity scale.

This result was already envisaged at kinetic transport equations written down by Northrop (1963), Kulsrud (1982) or Toptygin (1983). Thus Le Roux \& Webb (2007) clearly and correctly stated that the scatter-free version of this form of a transport equation necessarily implies the conservation of the particle magnetic moment $\mu$ in the plasma flow frame along the characteristics and fully contains the physics of particle mirroring.

\subsection{Some critical remarks on scattering terms}

Using the arguments presented above, we are also able to generalize these results to a few aspects of scattering terms appearing in the transport operator. Assuming that the scattering processes are elastic, no kinetic energy is transfered during the collisions, and $\boldsymbol{v}^{2}$ is an invariant. Then, for large inhomogenity scales, the magnetic moment is conserved as well, and the transport operator, even after including elastic scattering terms, should conserve the magnetic moment.

This result is slightly complicated for arbitrary inhomogenity scales. Since the magnetic field inducing the gyrational motion is always the local field at the current position of the ion, elastic scattering might conserve the kinetic energy, but not the magnetic moment, as a modification of the pitch angle $\xi$ clearly influences the magnetic moment $\mu \propto\left(1-\xi^{2}\right) v^{2}$.

On the other hand, one has to be really careful when studying Fokker-Planck-like scattering and diffusion terms, which only represent the first terms in a Taylor expansion of the magnetic field, which, may not be consistent with the source-free requirement for magnetic fields or similar electromagnetic boundary conditions. In a more accurate description, particle-wave interactions (which are the microphysical source of the scattering terms), are not a discrete, but a continuous process, which in principle must be treated fully consistent with Maxwell's equations. As demonstrated earlier in this study, taking the induced electric field into account allows to fully demonstrate the conservation of the magnetic moment. However, when these induced fields are not present, e.g. because one of the two electromagnetic field components is "weak" and, therefore, ignored, one must expect that the underlying equations do not describe a conservation of the magnetic moment, even when this conservation should be present. This might be able to explain why the conservation of the magnetic moment usually does not appear in modern numerical simulations.

\section{The magnetic moment in numerical simulations}

\subsection{Electron-proton shocks and the role of secondary shock components}

Shock simulations are a rather complicated, and in fact often physically untransparent, theoretical business. They, however, often are generally considered to give a self-consistent view of the plasma-particle interactions at shock structures. Here, we restrict ourselves to a careful overview of complications related to such a fully consistent modeling.

We begin with a (non-numerical) example demonstrating the subtle complications introduced with a more consistent modeling. We start with the classical, but nontrivial example of a quasineutral electron-proton system crossing an MHD shock wave. Due to the deceleration of the bulk plasma flow at the shock, there must be a local, spaceccharge-induced electric field present that decelerates the protons, while at the same time leads to an (initial) acceleration of the electrons. Taken together, these two effects generate a local space charge separation which in turn generates the electric field. All these effects must be present in a consistent numerical (or even analytical) description.

Now, we are not interested in the initial excitation of the shock wave. Instead, we aim to understand how existing numerical simulations handle the problem of quasineutrality, it's possible breaking and the reaction of gyrating ions to quasiinstantaneous magnetic transitions. In a recent paper on the MHD shock structure (Fahr et al. 2012b), we have shown that the electron overshooting in the downstream plasma frame is a highly important physical process that leads to strong electron heating, to entropy generation and to high compression ratios. As we have shown in that paper, the electron overshoot velocity in the downstream region is given by:

$\delta U_{\mathrm{e}}=\left|U_{2}-U_{1} \sqrt{1+\frac{m_{\mathrm{p}}}{m_{\mathrm{e}}}\left(1-\frac{1}{s^{2}}\right)}\right|$

where $U_{1,2}$ are the plasma bulk velocity at the upstream and downstream side of the shock, respectively.

From this formula, one evidently sees the importance of the mass ratio $m_{\mathrm{e}} / m_{\mathrm{p}}=\mu_{\mathrm{e}, \mathrm{i}}$ for the magnitude of the resulting overshoot velocity. For example, adopting a physically correct ratio of $\mu_{\mathrm{e}, \mathrm{i}}=1 / 1840$ will lead to

$$
\begin{aligned}
\delta U_{\mathrm{e}} & =\left|U_{2}-U_{1} \sqrt{1+\frac{m_{\mathrm{p}}}{m_{\mathrm{e}}}\left(1-\frac{1}{s^{2}}\right)}\right| \\
& \simeq\left(U_{1} / s\right)\left|1-\sqrt{\frac{m_{\mathrm{p}}}{m_{\mathrm{e}}}\left(s^{2}-1\right)}\right| \geq 43 U_{1} .
\end{aligned}
$$

On the other hand, adopting a smaller ratio, which is done by many numerical shock simulation studies, likewise results in a strongly different behavior. In the most extreme case, taking a highly artificial ratio of $\mu_{\mathrm{e}, \mathrm{i}}=1$ (i.e. like in an electron-positron pair plasma), this would yield a much lower value of

$\delta U_{\mathrm{e}}=\left|U_{2}-U_{2} \sqrt{1+s^{2}-1}\right|=U_{1}\left|\frac{1}{s}-1\right|=U_{1}\left(1-\frac{1}{s}\right) \leq U_{1}$.

This difference is sufficient to demonstrate that modern shock MHD simulations, which often use artificial values of this ratio $\mu_{\mathrm{e}, \mathrm{i}}$, thereby may react sensitively to the choice of this mass ratio, especially in handling the electron overshoot situation.

At the first look, it seems like that the ratio of the thermal energy $E_{\mathrm{t}, \mathrm{e}}$ of the electron overshoot and the kinetic energy $E_{\mathrm{kin}, \mathrm{i}}$ would be independent of the mass ratio $\mu_{\mathrm{e}, \mathrm{i}}$, which is given by

$$
\frac{E_{\mathrm{t}, \mathrm{e}}}{E_{\mathrm{kin}, \mathrm{i}}}=\frac{\frac{1}{2} m_{\mathrm{e}}\left(U_{1} \sqrt{\frac{m_{\mathrm{p}}}{m_{\mathrm{e}}}}\right)^{2} n_{2, \mathrm{e}}}{\frac{1}{2} m_{\mathrm{p}} U_{2}^{2} n_{2, i}}=s^{2} .
$$

However, the resulting compression ratio $s$ again strongly depends on the mass ratio. Making use of the formalism developed by Fahr et al. (2012b), we present (in Fig. 1) resulting compression ratios following the overshooting shock formalism, as a function of the mass ratio $\mu_{\mathrm{e}, \mathrm{i}}$ and the magnetic field strength. These results easily demonstrate that the compression ratio strongly depends on the electron-proton mass ratio. Therefore, any numerical simulation using unphysical mass ratios (which are typically found in the intervall $1 / 200<\mu_{\mathrm{e}, \mathrm{i}}<1$ ) might be flawed due to just this approximation. In addition, 


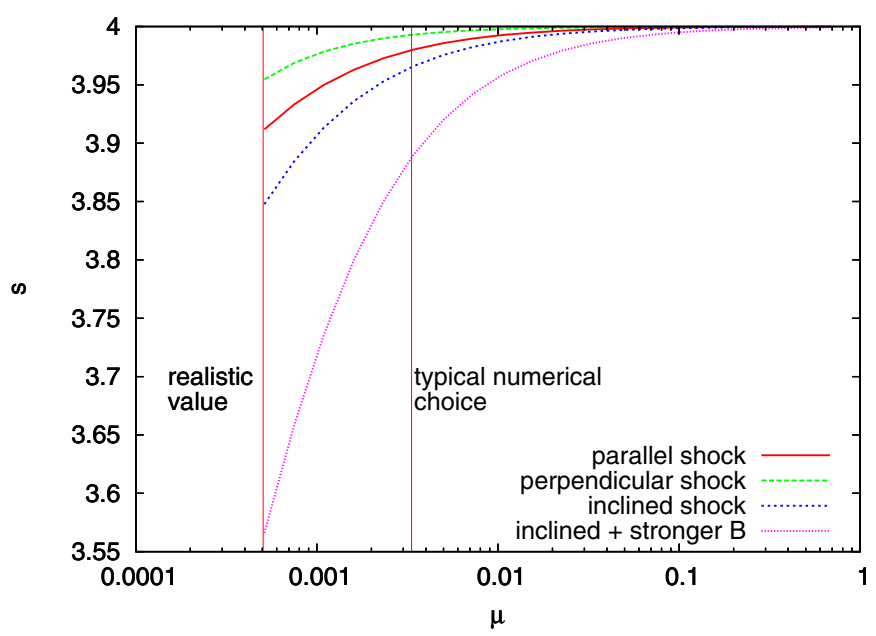

Fig. 1. Compression ratios obtained for a variety of shock inclinations and magnetic field strengths. Default magnetic field strength (first three curves) corresponds to a Parker field at $90 \mathrm{AU}$, the fourth line enhances the field by a factor of 2 .

whether this contribution is actually pronounced depends on the magnetic field strength, which, in the case of the solar wind termination shock, decreases with distance. Therefore, one must expect that the relative strength of the overshooting effect depends on the position of the shock system being studied. For example, the solar wind termination shock has a solar distance between approximately 90 and $200 \mathrm{AU}$, while interplanetary shock waves experience much stronger magnetic fields.

In the following section, we study selected properties of numerical simulations and estimate their respective potential for conflicts with a correct treatment of the magnetic moment.

\subsection{Hybrid shock simulations}

\subsubsection{Characteristic length- and timescales}

The main complication appearing in a numerical treatment of underlying electrodynamic processes appears with counting times in units of ion gyroperiods $\tau_{i}$, i.e. of $\Omega_{\mathrm{p}}^{-1}$, and measuring distances in units of inertial lengths given by $l_{\mathrm{i}}=v_{\mathrm{A}} / \Omega_{\mathrm{p}}$ (e.g. see a review by Lembege et al. 2004). This property is closely related to the magnetic moment conservation studied earlier here, as the magnetic moment is, essentially, equivalent to gyrational motion.

Looking to the fact that both ion density and magnetic field magnitude change from upwind to downwind, and also at timeand space-variable transient structures during the run of the simulation, thus means that these units are variable as well. Concerning upstream/downstream conditions, they change by

$\tau_{\mathrm{i} \text {,up }} / \tau_{\mathrm{i} \text {,down }}=B_{\text {down }} / B_{\text {up }} \simeq s$

and

$l_{\mathrm{i}, \text { up }} / l_{\mathrm{i}, \text { down }}=\left(v_{\mathrm{A}} / \Omega_{\mathrm{p}}\right)_{\mathrm{up}} /\left(v_{\mathrm{A}} / \Omega_{\mathrm{p}}\right)_{\mathrm{down}} \simeq \sqrt{s}$,

where $s$ denotes the usual MHD compression ratio defined as

$s=\frac{n_{\text {down }}}{n_{\text {up }}}=\frac{B_{\mathrm{t}, \text { down }}}{B_{\mathrm{t} \text {,up }}}$.

This means that both time- and space-resolution usually are much coarser on the upwind side compared to the downwind side in these simulations.
In addition, if one were to consider, individually, the specific length scales of both electrons and protons, the electrons would require a significantly higher resolution than the protons, introducing an additional complication. In most numerical simulations, the time scale problem is addressed by selecting an electron-to-proton mass ratio that reduces the differences in characteristic length scales. Other numerical situations treat electrons as a background fluid, which may eliminate the electron overshooting effect discussed earlier, and likewise result in a contradiction.

\subsubsection{Reference frames}

Another complication that appears in a numerical simulation is the choice of the reference frame in which specific plasma processes are considered. Typically, dispersion relations describing plasma waves and nonlinear particle-wave interactions are formulated in the reference frame of the bulk flow of the background plasma. The latter, however, in these simulations is an undefined frame due to the resulting time- and space-variable bulk velocities occuring during the run of the simulation. The usual Alfvénic wave mode and cyclotron resonances (see e.g. Scholer 1993, 1995; Scholer \& Kucharek 1999) are thus associated with a highly variable magnetic field and Alfvén velocity propagating in a highly variable plasma bulk frame. What in fact then counts for the study of ion-ion instabilities and creation of diffuse upstream ions is the relative ion motion with respect to resonant Alfvén modes. It is hard to imagine how hereby a satisfactory degree of consistency can be achieved.

\subsubsection{The electron-proton mass ratio}

Furthermore, most simulations are run as hybrid simulations, meaning that electrons are treated as massless fluids while ions are treated kinetically as particles. This means that a consistent value of electrical currents in such a code can only be achieved if the hydrodynamic value of the electron current $\boldsymbol{j}_{\mathrm{e}}=-e n_{\mathrm{e}} \boldsymbol{U}_{\mathrm{e}}$ is determined in a comparable accuracy as the kinetically determined ion current given by $j_{\mathrm{i}}=+e \sum \mathrm{d} n_{\mathrm{i}} \boldsymbol{v}_{\mathrm{i}}$, while the total electric current is determined by solving Maxwell's equation $\boldsymbol{\nabla} \times \boldsymbol{B}=4 \pi \boldsymbol{j}$. In order to reach high enough statistics to safely define electrical currents in a box of size $l_{\mathrm{i}}^{3}$, one either has to go to huge particle numbers in relevant time units $\tau_{\mathrm{i}}$, or to huge box sizes, i.e. resulting in a correspondingly coarse current resolution.

Faced with these problems (and limited computational resources), one commonly finds the usual restriction to a 4-d phasespace, i.e. only one space dimension $x$ is admitted, which means that the total current $j$ is restricted to one direction perpendicular to $\boldsymbol{x}$ given by

$j_{y}=-\frac{c}{4 \pi} \frac{\partial^{2}}{\partial x^{2}} A_{y}$

where $\boldsymbol{A}=\boldsymbol{A}_{y}$ is the magnetic vector potential (see Sgro \& Nielson 1976; Leroy et al. 1982 or Liewer et al. 1993).

Furthermore, these codes usually assume strict electric charge neutrality, meaning that the first moments of the electron and ion distribution functions are taken to be identical, i.e. $n_{\mathrm{e}}=n_{\mathrm{i}}$. However, seen in the frozen-in field frame the shock is induced by an electrostatic ramp connected with electric space charges $\eta$, i.e. $n_{\mathrm{e}}<n_{\mathrm{i}}$, that as a first step decelerates the ions as it accelerates the electrons and thereby consistently creates the required space charges (see Verscharen \& Fahr 2008). During the action of this space-charge-induced electric 
field $E_{\eta}=-\operatorname{grad} \Phi_{\eta}=e \int_{0}^{x}\left(n_{\mathrm{e}}-n_{\mathrm{i}}\right) \mathrm{d} x^{\prime}$, which is not taken into account in shock simulations, the inertial forces at ions and electrons are on the same order of magnitude $\left|m_{\mathrm{e}, \mathrm{i}} \frac{\mathrm{d} v_{\mathrm{e}, \mathrm{i}}}{\mathrm{d} t}\right| \simeq\left|e E_{\eta}\right|$, while in all simulations where space charges are not admitted the intertial forces acting on electrons are simply neglected because of their small masses.

Because of this reason, simulations using the commonly found quasineutrality condition, i.e. without actively studying the behavior of electrons, are inherently unable to control the conservation of the magnetic moment.

\subsubsection{Initial and boundary conditions}

Another point of a puzzling astonishment is connected with the initial conditions from which these simulations are generally started. Nearly all of these simulations (see e.g. Leroy et al. 1982; Leroy 1984; Liewer et al. 1993; Omidi et al. 1990; Scholer 1993, 1995) are carried out in a so-called "downstream box" which by itself defines the frame of the downstream plasma bulk flow. To initialize the simulation upstream ions are then injected from the left side of the box and are elastically reflected at the right border of the simulation box. This primarily induces a symmetrical ion counterflow situation and clearly would disturb the initial injection conditions at the left border, if the equation of ion motion in these simulations would not contain a friction term $\Pi$ describing an isotropisation of the counterflowing ions.

This term was originally understood as anomalous resistivity due momentum transfered from electrons to ions by means of Coulomb collisions. This type of friction of course only works under conditions of dense plasmas (see Sgro \& Nielson 1976; Leroy et al. 1982). For space plasmas characterized by small densities this term has meanwhile been understood as due to ion-resonant conditions with respect to plasma waves (Krauss-Varban \& Omidi 1991, 1995; Scholer 1995). However, no satisfactory consistency with respect to the description of this term has been achieved up to now, and therefore, existing numerical simulations of MHD shock waves are not self-consistent with respect to microphysical forces acting on the individual ions. This means that simulations of shock waves carried out using the "downstream box" initial boundary conditions can not be used to study, reliably and consistently, the (non-)conservation of the magnetic moment.

\subsubsection{Reaching an asymptotic state}

One classical problem with numerical simulations is the time required to reach a stable asymptotic state. Typically, this time directly scales with the quality of the initial conditions, i.e. the less the starting conditions deviate from the final state, the faster and more reliably does the code converge. Therefore, most simulations typically start with initial conditions derived from upstream values for the moments of the ion distribution (e.g. density and pressure) that have been derived using the usual MHD RankineHugoniot fluid relations. This approach implicitly assumes adiabatic pressure reaction with polytropic index $\gamma=5 / 3$ and a frozen-in magnetic field (see Leroy et al. 1982). This a priori prescription of a polytropic shock behavior therefore assumes the following relation between upstream (index 1) and downstream (index 2) ratios of the thermal to the magnetic pressure, or of upstream and downstream beta-values for the perpendicular shock:

$\beta_{2 \perp}=\frac{P_{2}}{\left(B_{2}^{2} / 8 \pi\right)}=\frac{P_{1}\left(\frac{n_{2}}{n_{1}}\right)^{\gamma}}{\left(B_{1}^{2} / 8 \pi\right)\left(n_{2} / n_{1}\right)^{2}}=\beta_{1} \cdot\left(\frac{n_{2}}{n_{1}}\right)^{\gamma-2}=\beta_{1} \cdot(s)^{-1 / 3}$.
Hence in view of compression ratios $s \leq 4$ there is expected a slight decrease of the plasma $\beta$ at the plasma passage from upstream to downstream by a factor of roughly 0.6 . For the case of the parallel shock $(\alpha=0)$ one would obtain instead:

$\beta_{2 \|}=\frac{P_{2}}{\left(B_{2}^{2} / 8 \pi\right)}=\frac{P_{1}\left(\frac{n_{2}}{n_{1}}\right)^{\gamma}}{\left(B_{1}^{2} / 8 \pi\right)}=\beta_{1} \cdot\left(\frac{n_{2}}{n_{1}}\right)^{\gamma}=\beta_{1} \cdot(s)^{\gamma}$.

In comparison to this expectation from which practically all shock simulations start, an alternative behavior is obtained for ions conserving their magnetic moments $\mu=m v_{\perp}^{2} / 2 B$ at the shock passage. Starting from Liouville's theorem of continuous differential phasespace flows applied to ions with isotropic distribution functions passing from upstream to downstream of the shock one obtains (see Fahr \& Siewert 2011, Eq. (27)):

$2 \pi U_{1} f_{1}\left(v_{1}\right) v_{1}^{2} \mathrm{~d} v_{1} \mathrm{~d} \cos \beta_{1}=2 \pi U_{2} f_{2}\left(v_{2}, \beta_{2}\right) v_{2}^{2} \mathrm{~d} v_{2} \mathrm{~d} \cos \beta_{2}$,

where $\beta_{1,2}$ are magnetic pitch angles of the upstream and downstream ion velocity space. After some elementary algebra, which we will not repeat here in full, this can be converted into (Fahr et al. 2012b)

$$
\begin{aligned}
P_{2} & =2 \pi(m / 2) \int f_{2}\left(v_{2}, \beta_{2}\right) v_{2}^{4} \mathrm{~d} v_{2} \mathrm{~d} \cos \beta_{2} \\
& =2 \pi(m / 2)\left(U_{1} / U_{2}\right) \int f_{1}\left(v_{1}\right) v_{1}^{2} v_{2}^{2} \mathrm{~d} v_{1} \mathrm{~d} \cos \beta_{1},
\end{aligned}
$$

with $P_{2}$ denoting the downstream thermal ion pressure, and furthermore then leads to (for a definition of the functions $A(\alpha)$ and $B(\alpha)$, see Fahr \& Siewert 2010)

$$
\begin{aligned}
P_{2}= & 2 \pi(m / 2)\left(U_{1} / U_{2}\right) \\
& \times \int f_{1}\left(v_{1}\right) v_{1}^{4}\left[\sin ^{2} \beta_{1} A(\alpha)+\cos ^{2} \beta_{1} B(\alpha)\right] \mathrm{d} v_{1} \mathrm{~d} \cos \beta_{1} .
\end{aligned}
$$

Assuming that the ion distribution function on the upstream side is pitchangle isotropic, the integrals with respect to $v_{1}$ and $\beta_{1}$ become independent of eachother, yielding

$$
\begin{aligned}
P_{2}= & 2 \pi(m / 2)\left(U_{1} / U_{2}\right) \int^{v} f_{1}\left(v_{1}\right) v_{1}^{4} \mathrm{~d} v_{1} \\
& \times \int^{\beta_{1}}\left[\sin ^{2} \beta_{1} A(\alpha)+\cos ^{2} \beta_{1} B(\alpha)\right] \mathrm{d} \cos \beta_{1}
\end{aligned}
$$

which can be evaluated to

$P_{2}=P_{1} \cdot s \cdot \frac{1}{2} \int^{\beta_{1}}\left[\sin ^{2} \beta_{1} A(\alpha)+\cos ^{2} \beta_{1} B(\alpha)\right] \mathrm{d} \cos \beta_{1}$,

where $P_{1}$ logically denotes the upstream thermal ion pressure. Ultimately, this expression then transforms into

$P_{2}=P_{1} \cdot s \cdot\left[\frac{2}{3} A(\alpha)+\frac{1}{3} B(\alpha)\right]$,

which allows one to relate the upstream and downstream pressures. This solution is different from what one would obtain from the classical, adiabatic Rankine-Hugoniot equations.

For the commonly studied configuration of a perpendicular shock (i.e. $\alpha=\pi / 2)$, this simply transforms to $[(2 / 3) A(\alpha)+$ $(1 / 3) B(\alpha)]_{\pi / 2}=(2 / 3) s+(1 / 3)$ (see Fahr \& Siewert 2011), and results in the following equation connecting the plasma $\beta$-values on both sides of the shock:

$\beta_{2 \perp}=\frac{P_{2}}{\left(B_{2}^{2} / 8 \pi\right)}=\frac{\left(B_{1}^{2} / 8 \pi\right)}{\left(B_{2}^{2} / 8 \pi\right)} \beta_{1} \cdot s \cdot\left[\frac{2}{3} s+\frac{1}{3}\right]=\frac{2 s+1}{3 s} \beta_{1}$, 


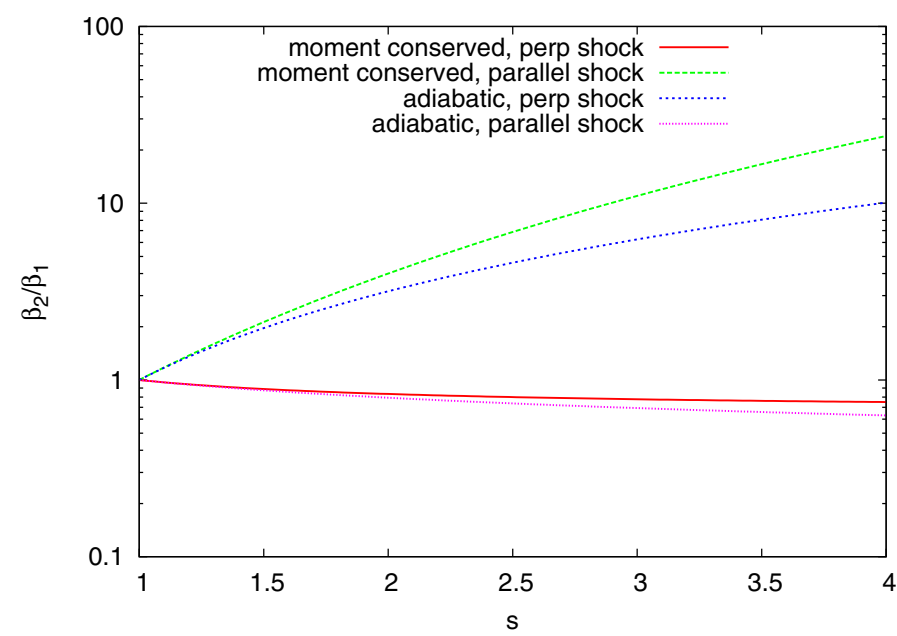

Fig. 2. Downstream plasma beta enhancement factor as a function of the MHD compression ratio for the magnetic moment-conserving approach and the adiabatic approach.

or, for the case of a parallel shock with $[(2 / 3) A(\alpha)+$ $(1 / 3) B(\alpha)]_{0}=(2 / 3)+(1 / 3) s^{2}$

$\beta_{2 \|}=\frac{P_{2}}{\left(B_{2}^{2} / 8 \pi\right)}=\frac{\left(B_{1}^{2} / 8 \pi\right)}{\left(B_{2}^{2} / 8 \pi\right)} \beta_{1} \cdot s \cdot\left[\frac{2}{3}+\frac{1}{3} s^{2}\right]=\frac{2 s+s^{3}}{3} \beta_{1}$.

To give an idea on the order of magnitude by which the plasma- $\beta$ can increase or decrease, we present a comparison between the magnetic moment-conserving relations (60 and 61) and the adiabatic relations (52 and 53) in Fig. 2. These results demonstrate that the difference between both approximations is on an order of 2, which can be sufficient to decide whether the downstream plasma is stable or unstable with respect to e.g. the mirror and firehose instabilities.

At the adiabatic jump, the plasma $\beta$-value in case of $\alpha=$ $\pi / 2$ is always decreased by values between $1 \geq s^{-1 / 3} \geq 0.6$ at the passage to the downstream side, while for $\alpha=0$ it is increased by a factor $s^{\gamma}$. In the case of the moment-conserving jump, and for the perpendicular shock $(\alpha=\pi / 2)$, the plasma beta is also decreased, however, by slightly less strong values between $1.0 \geq \frac{2 s+1}{3 s} \geq 0.75$, i.e. the magnetic energy always is preferentially increased with respect to the thermal energy at this shock passage. In the case of a parallel moment-conserving shock with $\alpha=0$, the downstream $\beta$-value is increased by strong factors between $1.0 \leq \frac{2 s+s^{3}}{3} \leq 24.0$.

To summarize this part of our study, we can point out that these differences should definitely impact the generation of different downstream instabilities that automatically appear at these types of shocks. These instabilities then must be treated in a fully consistent way, or one will almost certainly produce unstable downstream conditions, where no asymptotic boundary state is obtained. Interestingly enough, just this unstable downstream region is produced by the vast majority of simulation codes, which might be a hint that the electromagnetic conditions are not implemented correctly, and that it is consequently not possible to study the (non-)conservation of the magnetic moment with existing numerical methods.

In order to check the sensitivity of the shock simulation code with respect to MHD starting conditions, we could imagine it to be useful to start the run of the shock simulations from starting conditions that are provided by MHD shock relations derived under the requirement of conserved magnetic moments of ions and electrons at the passage of the plasma over the shock structure (see Fahr \& Siewert 2011). These relations deliver connections between downstream and upstream values of the fields, densities and temperatures clearly deviating from those relations elaborated on the basis of an adiabatic behavior of ion and electrons pressures. It might then be interesting to study whether these new initial starting conditions are closer or more distant from the asymptotic state found by the simulations compared to the conventional approach. Also it might be of interest whether the turbulent fluctuation amplitudes are different from the conventionally found ones and perhaps the asymptotic state is even different from the conventionally obtained one.

\section{Conclusions}

In this study, we have investigated the question of the conservation of the magnetic moment of ions gyrating in an arbitrary external magnetic field. We have also collected and expanded general properties of the magnetic moment, it's connection to the angular momentum and forces acting on the gyrocenter of the ion. This ultimately allowed us to understand the conservation of the magnetic moment in arbitrary situations. In general, a full electromagnetic treatment of the gyrating ion is required, which can not be carried out for the most general case; instead, numerical models are required to investigate this.

An innovative induction in our present approach is the introduction of overshoot kinetic energy at dealing with shockheated electrons (see Eqs. (44) through (44)). The induction of this physical process as was demonstrated by Fahr et al. (2012b) leads to strong nonadiabatic heating of electrons at their passage to the downstream side of the shock. This anomalous heating may be studied a little more critically in the light of more conventional approaches to describe shock-heated electrons. In a paper by Tokar et al. (1986) it was found from simulations that electrons are heated to significantly higher temperatures than that expected for adiabatic compression when the shock transition happens over only a few ion inertial lengths. As the authors state this heating cannot be due to cross field instabilities, but is due to direct electron acceleration in the cross shock electric field supplying overshoot kinetic energies. This form of heating seems to occur at HNM's (i.e. High Mach Number shocks) when upstream electrons can be considered as cold. In these simulations a mass ratio $\mu_{\mathrm{e}, \mathrm{i}}=m_{\mathrm{e}} / m_{\mathrm{i}}=10^{-2}$ is adopted, and we emphasize here that applying the correct mass ratio $\mu_{\mathrm{e}, \mathrm{i}}=5.4 \times 10^{-4}$ would lead to even stronger electron heating (see Eq. (45)). However, it is interesting to see that these authors find in the HNM-limit of shocks that electrons are energized such that the downstream thermal energy represents a large portion of the upstream kinetic ion ram energy, i.e.

$P_{\mathrm{e}, 2} \simeq \frac{3}{16}\left(\frac{1}{2} m_{\mathrm{i}} U_{1}^{2}\right) n_{\mathrm{e}, 2}=\frac{3 s}{16} P_{\mathrm{kin}_{\mathrm{i}, 1}} \leq \frac{3}{4} P_{\mathrm{kin}_{\mathrm{i}, 1}}$

a result, fairly similar to that presented in Eq. (47) of this work here. In a simulation study carried out more recently by Lembège et al. (2003), who were using a mass ratio $\mu_{\mathrm{e}, \mathrm{i}}=0.024$ it was meanwhile shown that the result showing over-adiabatic heating for electrons not only applies for HNM shocks, but is also found in shocks with moderate Mach numbers.

The results found by Schwartz et al. (1988) point into a similar direction. Investigating fast-mode collisionless shocks starting from Rankine-Hugoniot relations on the basis of an adiabatic heating with a polytrope $\gamma=5 / 3$, they recognize in their studies that downstream electrons appear to adapt to a much 
more effectively heated status with an effective polytropic index of $\gamma_{\text {eff }}=2.7$ through 3.0. They conclude that electron heating is nonadiabatic, but largely consistent with the conservation of the quantity $T_{\mathrm{e}, \perp} / B$, clearly pointing to something like the conservation of the elctron magnetic moment. This seems to indicate that our considerations with respect to the electron overshoot kinetic energy are not out of any rational context.

Following this theoretical study, we have investigated commonly performed numerical simulations and the approximations used by them. As we have demonstrated, there are (at least) five different aspects that prevent existing numerical situations from allowing one to study the conservation of the magnetic moment: 1) The small electron mass does not allow to study electrons and protons on a self-consistent unique length scale. 2) Most numerical simulations do not allow to define the correct reference frame used to study (electromagnetic) plasma-wave interactions. 3) The electon-to-proton mass ratio used by most simulations is artificially high and does not allow to reproduce a consistent electromagnetic behavior of the individual ions. 4) Many simulations of shock waves use boundary conditions that do not consistently allow to calculate microphysical forces acting on the individual ions. 5) Many existing numerical simulations do not seem to arrive at an asymptotically stable state.

Taking these arguments together, even if a specific code allows to overcome one or more of these underlying complications, we are not aware of any numerics that allows to eliminate all five of these aspects, and therefore, we have to come to the conclusion that no existing numerical plasma code is sufficiently consistent to study the (non-)conservation of the magnetic moment, at least under astrophysical conditions.

Acknowledgements. M. Siewert is grateful to the Deutsche Forschungsgemeinschaft for financial support granted to him in the frame of the project Si-1550/2-2.

\section{References}

Barakat, A. R., \& Schunk, R. W. 1983, J. Geophys. Res., 88, 7887

Barakat, A. R., \& Schunk, R. W. 1989, J. Geophys. Res., 94, 1487

Barakat, A. R., \& Barghouthi, I. A. 1994, Geophys. Res. Lett., 21, 2279

Barghouthi, I. A., Barakat, A. R., \& Schunk, R. W. 1994, Ann. Geophys., 12, 1076

Cary, J. R., \& Brizard, A. J. 2009, Rev. Mod. Phys., 81, 693

Chen, F. F. 1977, Introduction to Plasma Physics (New York: Plenum Press)

Earl, J. A. 1976, ApJ, 206, 301

Fahr, H.-J., \& Fichtner, H. 2011, A\&A, A92

Fahr, H., \& Scherer, K. 2004, Astrophysics and Space Sciences Transactions, 1, 3

Fahr, H.-J., \& Scherer, K. 2005, J. Geophys. Res., 110, A02103

Fahr, H.-J., \& Siewert, M. 2006, A\&A, 458, 13

Fahr, H.-J., \& Siewert, M. 2010, ASTRA, 6, 31
Fahr, H.-J., \& Siewert, M. 2011, A\&A, 527, A125

Fahr, H. J., Bird, M. K., \& Ripken, H. W. 1977, A\&A, 58, 339

Fahr, H.-J., Chashei, I. V., \& Siewert, M. 2012a, A\&A, 537, A95

Fahr, H.-J., Siewert, M., \& Chashei, I. 2012b, Ap\&SS, 341, 265

Giacalone, J., \& Jokipii, J. R. 2006, in Physics of the Inner Heliosheath, eds. J. Heerikhuisen, V. Florinski, G. P. Zank, \& N. V. Pogorelov, AIP Conf. Ser., 858, 202

Jackson, J. D. 1975, Classical electrodynamics

Krauss-Varban, D., \& Omidi, N. 1991, J. Geophys. Res., 96, 17715

Krauss-Varban, D., \& Omidi, N. 1995, Geophys. Res. Lett., 22, 3271

Kucharek, H., Möbius, E., \& Scholer, M. 2006, in Physics of the Inner Heliosheath, eds. J. Heerikhuisen, V. Florinski, G. P. Zank, \& N. V. Pogorelov, AIP Conf. Ser., 858, 196

Kucharek, H., Pogorelov, N., Moebius, E., \& Lee, M. A. 2010, in AIP Conf. Ser. 125, 1302, eds. J. Le Roux, G. P. Zank, A. J. Coates, \& V. Florinski

Kulsrud, R. M. 1982, Phys. Scripta T, 2, 177

Kunstmann, J. E. 1979, ApJ, 229, 812

Le Roux, J. A., \& Webb, G. M. 2006, in Physics of the Inner Heliosheath, eds. J. Heerikhuisen, V. Florinski, G. P. Zank, \& N. V. Pogorelov, AIP Conf. Ser., 858, 190

Le Roux, J. A., \& Webb, G. M. 2007, ApJ, 667, 930

Le Roux, J. A., Matthaeus, W. H., \& Zank, G. P. 2001, AGU Fall Meeting Abstracts, A731

Le Roux, J. A., Zank, G. P., Matthaeus, W. H., \& Milano, L. J. 2002, AGU Fall Meeting Abstracts, A11

Lembege, B., Giacalone, J., Scholer, M., et al. 2004, Space Sci. Rev., 110, 161

Lembège, B., Savoini, P., Balikhin, M., Walker, S., \& Krasnoselskikh, V. 2003, J. Geophys. Res., 108, 1256

Leroy, M. M. 1984, Adv. Space Res., 4, 231

Leroy, M. M., Winske, D., Goodrich, C. C., Wu, C. S., \& Papadopoulos, K. 1982, J. Geophys. Res., 87, 5081

Li, G., \& Zank, G. P. 2006, in Physics of the Inner Heliosheath, eds. J. Heerikhuisen, V. Florinski, G. P. Zank, \& N. V. Pogorelov, AIP Conf. Ser., 858, 183

Liewer, P. C., Goldstein, B. E., \& Omidi, N. 1993, J. Geophys. Res., 98, 15211

Litvinenko, Y. E., \& Schlickeiser, R. 2011, ApJ, 732, L31

Northrop, T. G. 1963, Rev. Geophys. Space Phys., 1, 283

Omidi, N., Quest, K. B., \& Winske, D. 1990, J. Geophys. Res., 95, 20717

Roelof, E. C. 1969, in Lectures in High-Energy Astrophysics, eds. H. Ögelman, \& J. R. Wayland, 111

Sarris, E. T., \& van Allen, J. A. 1974, J. Geophys. Res., 79, 4157

Schlickeiser, R. 2009, Mod. Phys. Lett. A, 24, 1461

Schlickeiser, R., \& Shalchi, A. 2008, ApJ, 686, 292

Scholer, M. 1993, J. Geophys. Res., 98, 47

Scholer, M. 1995, Adv. Space Res., 15, 125

Scholer, M., \& Kucharek, H. 1999, Ap\&SS, 264, 527

Schwartz, S. J., Thomsen, M. F., Bame, S. J., \& Stansberry, J. 1988, J. Geophys. Res., 93, 12923

Sgro, A. G., \& Nielson, C. G. 1976, Phys. Fluids, 19, 129

Siewert, M., \& Fahr, H.-J. 2008, A\&A, 485, 327

Spangler, S. R., \& Basart, J. P. 1981, ApJ, 243, 1103

Terasawa, T. 1979, Planet. Space Sci., 27, 193

Tokar, R. L., Aldrich, C. H., Forslund, D. W., \& Quest, K. B. 1986, Phys. Rev. Lett., 56, 1059

Toptygin, I. N. 1983, Cosmic rays in interplanetary magnetic fields (Norwell/Mass.: D. Reidel Publ.)

Verscharen, D., \& Fahr, H.-J. 2008, A\&A, 487, 723 